\title{
$\sigma, \kappa$ and $f_{0}(980)$ in E791 and BES II data
}

\author{
D.V. Bugg
}

Queen Mary, University of London, Mile End Rd., London E1 4NS, UK

\begin{abstract}
Both $\sigma$ and $\kappa$ are well established from E791 data on $D \rightarrow 3 \pi$ and $K \pi \pi$ and BES II data on $J / \Psi \rightarrow \omega \pi^{+} \pi^{-}$and $K^{+} K^{-} \pi^{+} \pi^{-}$. Fits to these data are accurately consistent with $\pi \pi$ and $K \pi$ elastic scattering when one allows for the Adler zero which arises from Chiral Symmetry Breaking. The phase variation with mass is also consistent between elastic scattering and production data.
\end{abstract}

PACS: 13.75Jx, 14.40.Cs, 1440.Ev

Keywords: mesons, resonances

At the conference, results on a large range of experiments on the $\sigma$ and $\kappa$ were presented. Here, available space limits the discussion to E791 and BES II data. Space also prevents a review of the large background of theoretical papers fitting elastic scattering. An extended review covering all these topics will appear on the web.
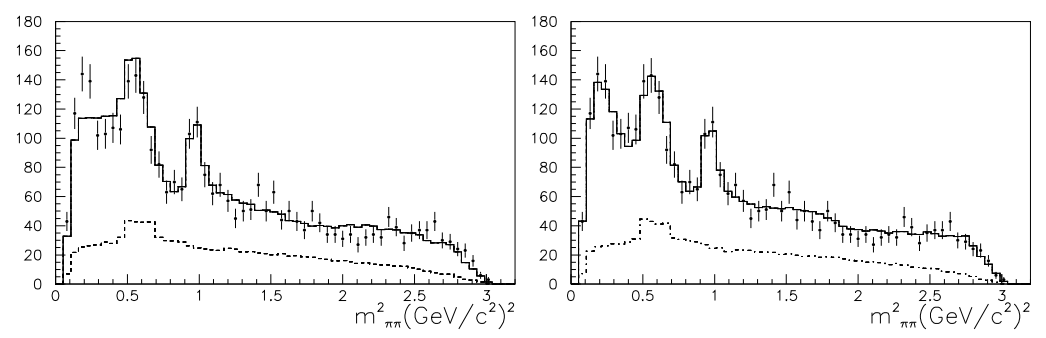

Figure 1: The $\pi \pi$ mass projection of E791 data for $D^{+} \rightarrow \pi^{+}\left(\pi^{-} \pi^{+}\right)$, (a) without, (b) with $\sigma$ in the fit. The dashed histogram shows background. 

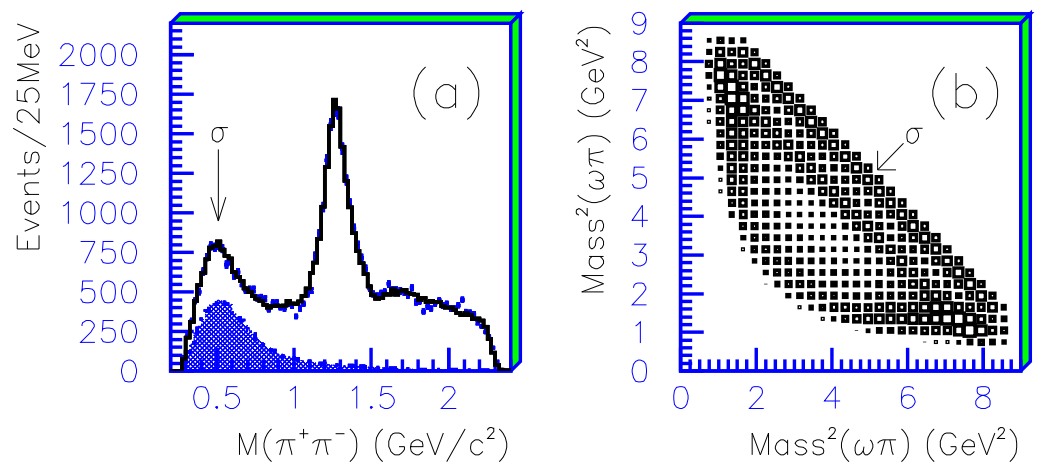

Figure 2: (a) The $\pi \pi$ mass projection of BES data; the histogram shows the fit and the hatched area the $\sigma$ contribution; (b) the Dalitz plot.

\section{The $\sigma$ pole}

Early evidence for the $\sigma$ pole came from elastic scattering data. Markushin and Locher [1] summarise many determinations. Renewed interest was sparked off by E791 data on $D^{+} \rightarrow\left(\pi^{+} \pi^{-}\right) \pi^{+}[2]$. The $\pi \pi$ mass projection, shown in Fig. 1, has a low mass peak. The fit assumed a conventional Breit-Wigner resonance with $\Gamma(s) \propto \rho(s)$, where $\rho(s)$ is Lorentz invariant phase space $2 k / \sqrt{s}=\sqrt{1-4 m_{\pi}^{2} / s}$ and $k$ is centre of mass momentum. This choice of $\Gamma(s)$ will later be shown to be inappropriate, but correcting it to a better form introduces only changes of detail. The pole position is shown in the first entry of Table 1 below. Oller's very interesting refit of these data with ChPT input is also shown there [3].

Fig. 2 shows higher statistics BES II data [4] for $J / \Psi \rightarrow \omega \pi^{+} \pi^{-}$. Dominant signals are $f_{2}(1270), b_{1}(1235)$ and $\sigma$, which is clearly visible as a flat band along the right-hand edge of the Dalitz plot in (b). The $0^{+}$contribution is shown shaded in (a). All four parametrisations which were tried are consistent with an average pole position $\mathrm{M}=(541 \pm 39)-\mathrm{i}(252 \pm 42) \mathrm{MeV}$.

Fig. 3(a) shows the intensity of $\pi \pi$ elastic scattering v. mass. The 

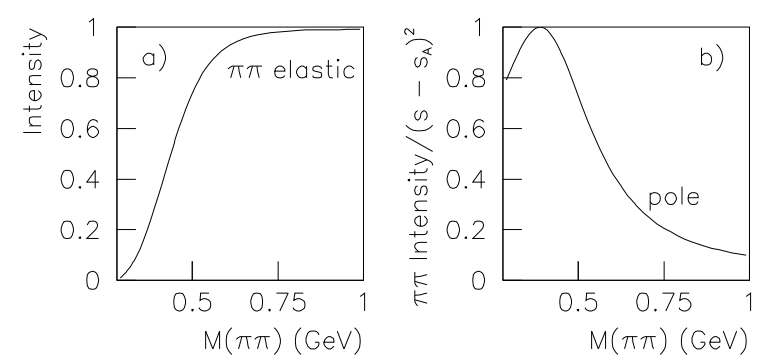

Figure 3: (a) The $\pi \pi$ intensity in elastic scattering, (b) with the Adler zero divided out.

obvious puzzle is why there is no low mass peak like that in production data of Fig. 2. There is a simple explanation, given in 1965-6 by Adler and Weinberg [5]. They proposed that massless $\pi$ of zero momentum have zero scattering amplitude. If the $I=0$ S-wave $\pi \pi \rightarrow \pi \pi$ amplitude is expanded as a power series $a m_{\pi}^{2}+b k^{2}$, consistency between $s, t$ and $u$ channels requires that the amplitude is proportional to $\left(s-0.5 m_{\pi}^{2}\right)$ and has a zero at the Adler point $s_{A}=0.5 m_{\pi}^{2}$. Fig. 3(b) shows the result of dividing (a) by $\left(s-s_{A}\right)^{2}$. Instantly one sees a resemblance with the $\sigma$ peak of Fig. 2. So the solution to the puzzle is that the matrix element for $\pi \pi$ elastic scattering is strongly $s$-dependent: a situation unlike most other resonances.

Let us write the elastic $\sigma$ amplitude as

$$
\begin{aligned}
T_{e l}^{00} & =[\eta \exp (2 i \delta)-1] / 2 i \\
& =\frac{N(s)}{D(s)}=\frac{N_{e l}(s)}{M^{2}-s-i N_{t o t}(s)} .
\end{aligned}
$$

Here $N_{e l}(s)$ is real for $s \geq 0$; the phase variation comes purely from the denominator $D(s)$. This denominator is universal for all processes involving a $\pi \pi$ pair. For elastic scattering, the Adler zero in $N(s)$ nearly cancels the $\sigma$ pole for low masses. However, the numerator $N(s)$ is not universal; it is quite different for production processes, where the left-hand cut is distant. Later, it will be shown that E791 data for $D \rightarrow(K \pi) \pi$ require $N(s)_{\operatorname{prodn}}=1$ 
within errors. The production amplitude will therefore be written

$$
T_{p r o d n}^{00}=\Lambda / D(s),
$$

where $\Lambda$ is a complex constant.
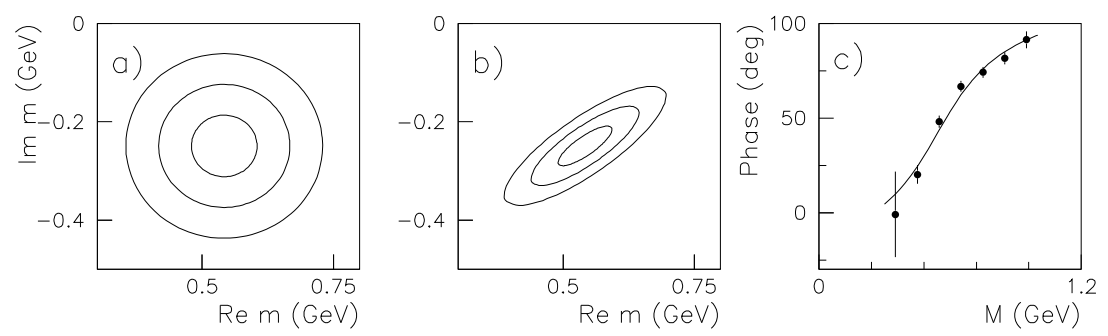

Figure 4: Contours of intensity for (a) production, (b) elastic scattering; (c) the phase of the $\sigma$ amplitude in mass bins $100 \mathrm{MeV}$ wide, compared with the global fit.

Fig. 4 illustrates contours of constant intensity for (a) production, (b) elastic scattering. In (b), the Adler zero suppresses the intensity near threshold. The elastic phase shift on the real $s$ axis (where experiments are done) reaches $90^{\circ}$ only at $M>900 \mathrm{MeV}$, far above the pole. It is this feature which confuses many people. The phase varies rapidly off the real axis because the width of the resonance increases with $s$.

Data from BES II, $K_{e 4}[6]$ and Cern-Munich [7] are fitted empirically with:

$$
N(s)=M\left(s-0.5 m_{\pi}^{2}\right) \exp \left[-\left(s-M^{2}\right) / A\right](1+\beta s) \rho_{\pi \pi}(s)+M \Gamma_{4 \pi}(s) .
$$

The exponential is required by $\pi \pi$ elastic data to cut off $N(s)$ above $1 \mathrm{GeV}$. The term $M \Gamma_{4 \pi}$ fits inelasticity above $1 \mathrm{GeV}$, but has little effect on the $\sigma$ pole. Note from Fig. 2(a) that the $\sigma$ intensity fitted to BES data is small above $1 \mathrm{GeV}$. The $\sigma$ pole is therefore distinct from the broad $f_{0}(1535)$ fitted by Anisovich and Sarantsev [8]. It is also distinct from the broad pole fitted around $1 \mathrm{GeV}$ by Au, Morgan and Pennington [9]. Contributions from 


\begin{tabular}{|c|c|c|c|}
\hline State & Reference & Data & $\begin{array}{l}\text { Pole position } \\
\quad(\mathrm{MeV})\end{array}$ \\
\hline \multirow[t]{6}{*}{$\sigma$} & {$[2]$} & $D^{+} \rightarrow\left(\pi^{+} \pi^{-}\right) \pi^{+}$ & $(489 \pm 26)-i(173 \pm 26)$ \\
\hline & {$[3]$} & $D^{+} \rightarrow\left(\pi^{+} \pi^{-}\right) \pi^{+}$ & $470-i 220$ \\
\hline & {$[4]$} & $J / \Psi \rightarrow \omega\left(\pi^{+} \pi^{-}\right)$ & $(541 \pm 39)-i(252 \pm 42)$ \\
\hline & [10] & $\pi \pi \rightarrow \pi \pi$ & $(470 \pm 30)-i(295 \pm 20)$ \\
\hline & [13] & $\pi \pi \rightarrow \pi \pi$ & $445-i 221$ \\
\hline & {$[25]$} & $\pi \pi \rightarrow \pi \pi$ & $(470 \pm 50)-i(285 \pm 50)$ \\
\hline \multirow[t]{10}{*}{$\kappa$} & {$[28]$} & $D^{+} \rightarrow\left(K^{-} \pi^{+}\right) \pi^{+}$ & $(721 \pm 61)-i(292 \pm 131)$ \\
\hline & {$[30]$} & $J / \Psi \rightarrow K^{+} \pi^{-} K^{-} \pi^{+}$ & $(760 \pm 41)-i(420 \pm 75)$ \\
\hline & [29] & $J / \Psi \rightarrow K^{+} \pi^{-} K^{-} \pi^{+}$ & $(841 \pm 82)-i(309 \pm 87)$ \\
\hline & {$[33]$} & $K \pi \rightarrow K \pi$ & $(722 \pm 60)-i(386 \pm 50)$ \\
\hline & here & all & $750_{-55}^{+30}-i(342 \pm 60)$ \\
\hline & {$[3]$} & $D^{+} \rightarrow\left(K^{-} \pi^{+}\right) \pi^{+}$ & $710-i 310$ \\
\hline & {$[12]$} & $K \pi \rightarrow K \pi$ & $770-i(250-425)$ \\
\hline & {$[14]$} & $K \pi \rightarrow K \pi$ & $708-i 305$ \\
\hline & {$[15]$} & $K \pi \rightarrow K \pi$ & $753-i 235$ \\
\hline & {$[24]$} & $K \pi \rightarrow K \pi$ & $(594 \pm 79)-i(362 \pm 322)$ \\
\hline \multirow[t]{2}{*}{$f_{0}(980)$} & [34] & $J / \Psi \rightarrow \phi \pi^{+} \pi^{-}$ & $(998 \pm 4)-i(17 \pm 4)$ \\
\hline & {$[12]$} & $\pi \pi \rightarrow \pi \pi$ and $K K$ & $994-\mathrm{i} 14$ \\
\hline$a_{0}(980)$ & {$[35]$} & $\bar{p} p \rightarrow \eta \pi \pi$ and $\omega \eta \pi^{0}$ & $(1036 \pm 5)-i(84 \pm 9)$ \\
\hline
\end{tabular}

Table 1: Summary of pole positions.

$f_{0}(980), f_{0}(1370)$ and $f_{0}(1500)$ to elastic scattering are included by multiplying their S-matrices with $S_{\sigma}$, to satisfy unitarity; i.e. their phases add. For production, there are hundreds of open channels for $D$ and $J / \Psi$ decays. Within individual channels, unitarity plays a negligible role. Following the standard isobar model, amplitudes are added using a complex coupling constant $\Lambda=g \exp \left(i \phi_{0}\right)$ for each amplitude.

The $K_{e 4}$ data are available up to $380 \mathrm{MeV}$ and there is then a gap in elastic data until $560 \mathrm{MeV}$, where Cern-Munich data begin. The $\sigma$ pole lies in the mass range where there are no elastic data. Although this gap may be bridged by using dispersion relations, the production data are obviously important in filling the gap directly. 
In the BES data, $b_{1} \pi$ contributes $41 \%$ of the intensity and $\sigma \omega 19 \%$. Strong interferences between them determine the phase variation of the $\sigma$ with mass. The data have been divided into bins $100 \mathrm{MeV}$ wide from 400 to $1000 \mathrm{MeV}$. Unfortunately, the $b_{1}$ band runs off the corner of the Dalitz plot and does not interfere significantly with the $\sigma$ below $400 \mathrm{MeV}$. Fig. 4(c) shows phases for individual bins, in good agreement with the global fit.

\section{$1.1 \quad$ Theory}

Colangelo, Gasser and Leutwyler [10] have made a precise determination of the $\sigma$ pole from elastic data and $K_{e 4}$ without using production data. This work has the virtue of fitting both the physical region and the left-hand cut.

Oset, Oller, Pelaez and collaborators fit elastic data using 'unitarised' Chiral Perturbation Theory [11-16]. They use ChPT for lowest order and take rescattering from the next order. They fit successfully not only the $I=0 \mathrm{~S}$-wave, but also the repulsive $I=2 \mathrm{~S}$-wave. This implies that they fit the nearby left-hand cut correctly as well as the physical region.

Schechter's group has also contributed a series of papers on all of $\sigma, \kappa$, $f_{0}(980)$ and $a_{0}(980)[17-21]$. This work examines possible mixing between 2-quark and 4-quark states. Zheng and collaborators at Peking University have developed new types of dispersion relations and have applied them to analysis of data on the $\sigma$ and $\kappa[22-25]$.

Van Beveren and Rupp have an interesting but quite different scheme, modelling the spectrum and decays of all mesons from the lightest to charmonium and bottomonium states. This is done with a harmonic oscillator [26] or an arbitrary confining potential [27]. They allow for decays by coupling q-qbar states to outgoing mesons through a transition potential. In this scheme, $f_{0}(1370), a_{0}(1450)$, etc. are regular but unitarised $q \bar{q}$ states; $\sigma$,

$\kappa, f_{0}(980)$ and $a_{0}(980)$ appear as 'extra' states created by coupling of $q \bar{q}$ to decay channels [26,27].

\section{The $\kappa$ pole}

E791 data on $D^{+} \rightarrow\left(K^{-} \pi^{+}\right) \pi^{+}$provided the first evidence for the $\kappa$ pole from production data [28]. A combined fit to these and other data will be considered in detail below. 

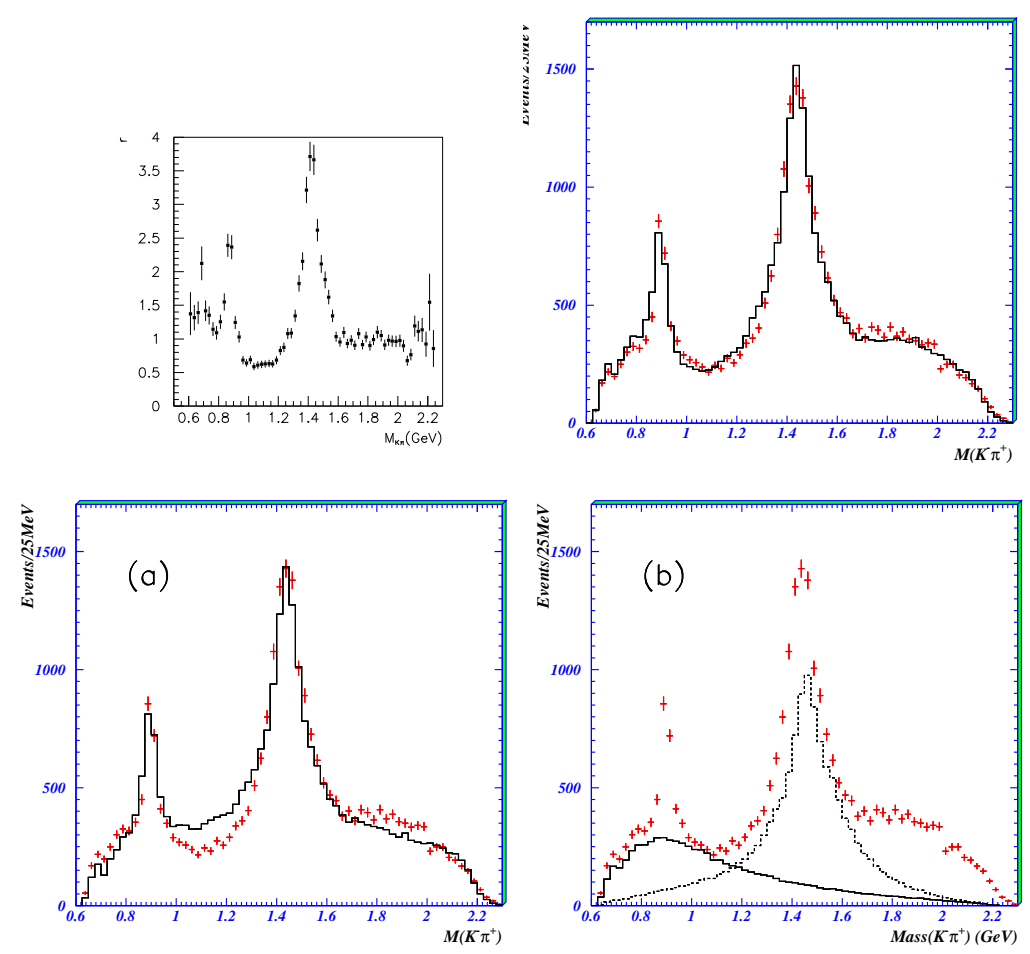

Figure 5: BES II data on the $\kappa$. Upper left: the $K \pi$ S-wave spectrum in $J / \Psi \rightarrow K^{*}(890)(K \pi)_{S}$ after factoring out phase space; upper right: the fit to $m(K \pi)$; (a) the bad fit if the Adler zero is omitted; (b) the $\kappa$ contribution (full histogram) and $K^{0}(1430)$ (dashed). 
Next, BES II data on $J / \Psi \rightarrow K^{+} \pi^{-} K^{-} \pi^{+}$reveal the $\kappa$ in $J / \Psi \rightarrow$ $K^{*}(890) \kappa[29,30]$. If one selects a $K^{ \pm} \pi^{\mp}$ pair within $50 \mathrm{MeV}$ of $890 \mathrm{MeV}$, the accompanying $K^{\mp} \pi^{ \pm}$pair has the mass projection shown in Fig. 5(a) after dividing out phase space. The broad low mass peak below the $K^{*}(890)$ is the evidence for the $\kappa$; one point has a rather large statistical fluctuation. Full details of my fit to these data are given in Ref. [30]. Other strong components are $K^{*}(890) K_{0}(1430), K^{*}(890) K_{2}(1430), K_{0}(1430) K_{0}(1430)$ and $K K_{1}(1270)+K K_{1}(1400)$, followed by $K_{1} \rightarrow K^{*} \pi$ and $\rho K$. Since $K_{1}$ decays populate the low mass $K \pi$ range, it is essential to demonstrate that $K_{1}$ decays do not reproduce the $\kappa$ peak.

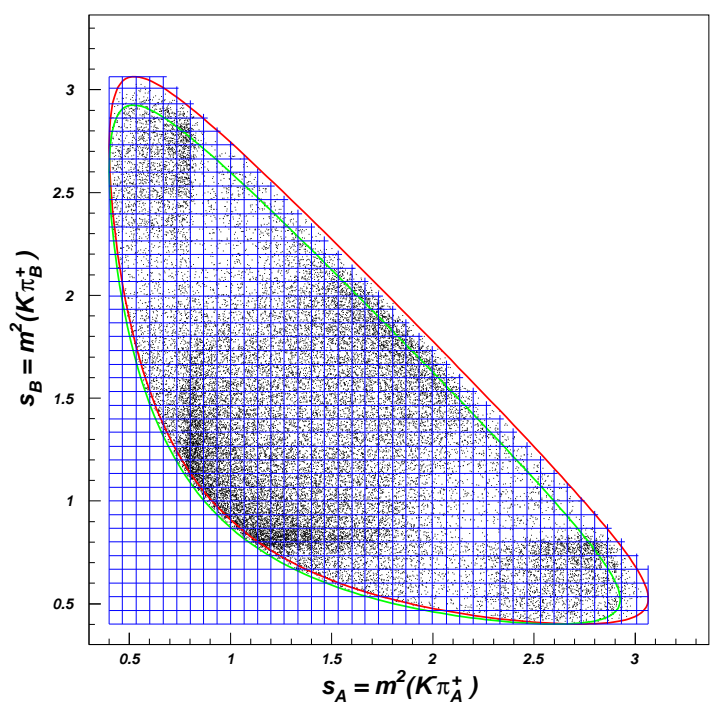

Figure 6: The Dalitz plot for E791 data for $D^{+} \rightarrow K^{-} \pi^{+} \pi^{+}$.

A combined fit is made to BES and LASS data [31] for the $K \pi I=1 / 2$ S-wave. The fit uses eqn. (1) with

$$
N(s)=M\left(s-s_{A}\right) \exp (-\alpha \sqrt{s}) \rho_{K \pi}(s)
$$

and $s_{A}=m_{K}^{2}-0.5 m_{\pi}^{2}$. The fit to the raw $K \pi$ mass projection is shown in Fig. 5(b). If the factor $\left(s-s_{A}\right)$ is omitted, the poor fit is shown in Fig. 
$5(\mathrm{c})$. The $\kappa$ and $K_{0}(1430)$ mass projections are shown in Fig. $5(\mathrm{~d})$. There is destructive interference between them. Sensitivity to this interference is one reason for fitting the LASS data simultaneously. A second point is to show that eqn. (4) fits both sets of data successfully.

The phase variation of the $\kappa$ with mass is well determined in two ways which agree. Firstly, there is large interference between channels $K^{*}(980) \kappa$ and $K K_{1}(1270+1400)$. Secondly, there is a large interference between $\kappa$ and $K_{0}(1430)$, which both contribute to the $K \pi$ S-wave. A bin-by-bin fit has been made where the $\kappa$ signal is fitted in magnitude and phase in 10 individual bins $100 \mathrm{MeV}$ wide. Results are shown in Fig. 8(c) below.

An independent analysis of exactly the same data has been reported by the BES group [29]. This fit omits interferences of the $\kappa$ with both $K_{0}(1430)$ and the $K_{1}$ 's. It therefore provides no information on the phase of the $\kappa$. It also does not fit $K^{*}(890)$ decays; angular correlations with these decays are crucial in separating channels $K^{*}(890) K_{0}(1430)$ and $K^{*}(890) K_{2}(1430)$.

\section{Re-analysis of E791 data}

The Dalitz plot for E791 data in shown in Fig. 6. There is obvious interference between $K^{*}(890)$ and the $K \pi$ S-wave, creating an asymmetry around the $K^{*}$ band. A new fit has been reported recently where the magnitude and phase of the $K \pi$ S-wave anplitude is fitted separately in 37 mass bins [32]. Magnitudes and phases are shown below in Figs. 7 and 8 . No attempt is made to separate $\kappa$ and $K_{0}(1430)$.

Both E791 and LASS data contain fairly weak $K_{0}(1430)$ structures. Results depend the variation of $\kappa$ intensity under the $K_{0}(1430)$; i.e. the width of the $\kappa$ is correlated with the fit to $K_{0}(1430)$. I have made a combined fit to the data of LASS, BES and E791. The BES data define well the $K_{0}(1430)$ peak, which is much more conspicuous than in either LASS or E791 data.

In the E791 fit (and also Ref. [28]), the amplitude includes a production form factor $F=\exp \left(-\alpha q^{2}\right)$, where $q$ is the momentum of the $\kappa$ in the $D$ rest frame and $\alpha=2.08 \mathrm{GeV}^{-2}$. I have varied $\alpha$ and Fig. 7 shows results. Panel (a) uses $\alpha=0$ and gives the best fit. In (b)-(d) $\alpha$ increases in equal steps to the E791 value in (d). Within errors, $\alpha$ optimises at 0. This corresponds to a point-like decay $D \rightarrow \kappa \pi$ with an RMS radius $<0.38$ fm with $95 \%$ confidence. Fits to the phase of the $\kappa$ also optimise at $\alpha=0$. 

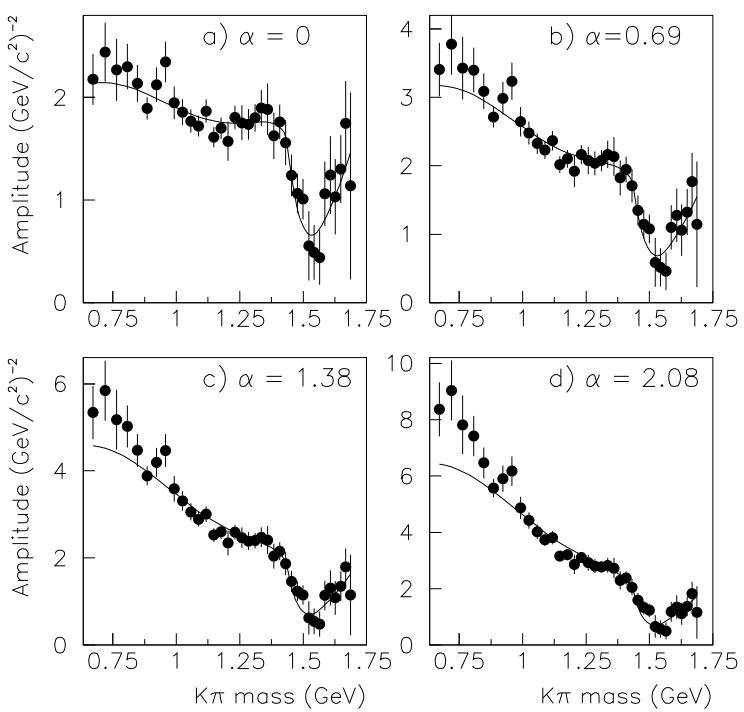

Figure 7: The fit to the magnitude of the $K \pi$ S-wave in E791 data for four values of $\alpha$ in the production form factor.

The combined fit gives a $\kappa$ pole at $\mathrm{M}=\left(750_{-55}^{+30}\right)-\mathrm{i}(342 \pm 60) \mathrm{MeV}$. This compares with $\mathrm{M}=(722 \pm 60)-\mathrm{i}(386 \pm 50) \mathrm{MeV}$ for LASS data alone [33] and $\mathrm{M}=(760 \pm 20 \pm 40)-i(420 \pm 45 \pm 60) \mathrm{MeV}$ for LASS + BES data only [30]. It reduces errors and brings all three sets of data into close agreement.

Fits to the BES and LASS data are shown in Fig. 8. Figs. 8(a) and (b) show the fit to LASS data; (c) and (d) show that the fit to the magnitude and phase of the $\kappa$ in BES data agrees. Again, the phase variation with mass agrees between elastic scattering and production data. This demonstrates that both can be fitted with the same $D(s)$, and therefore that the data can be fitted with a resonance alone without the need for any background amplitude. The $K_{0}(1430)$ is a large signal in BES data with well defined centroid and width. The fit to this peak is shown in Fig. 8(e) using 25\% $K^{*}(890) K_{2}(1430)$ and $75 \% K^{*}(890) K_{0}(1430)$, as determined from my fit to BES data; (f) shows the good fit to E791 phases using $\alpha=0$. 

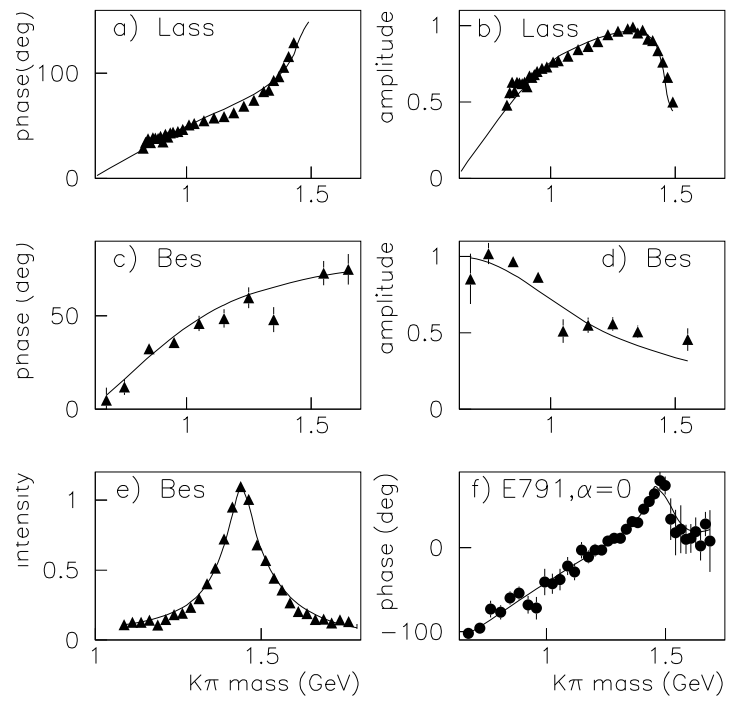

Figure 8: Fits to (a) Lass phases for $K \pi$ elastic scattering; (b) magnitudes of the Lass elastic scattering amplitude; (c) the phase of the $\kappa$ signal in BES II data after dividing into $100 \mathrm{MeV}$ mass bins, (d) BES magnitudes in individual bins, (e) the magnitude of the $K_{0}(1430)$ peak above background in BES data, (f) E791 phases with $\alpha=0$.

\section{$4 \quad f_{0}(980)$ and $a_{0}(980)$}

An excellent new determination of the parameters of $f_{0}(980)$ is obtained from BES data on $J / \Psi \rightarrow \phi \pi^{+} \pi^{-}$and $\phi K^{+} K^{-}$[34]. The $f_{0}(980)$ appears as a strong peak in $\pi \pi$ and a smaller but clear peak in $K K$. From the relative numbers of events in these two peaks, the ratio $g^{2}(K K) / g^{2}(\pi \pi)$ is well determined. Previously results for this ratio have been erratic. The pole position is shown in Table 1, together with the best available pole position for $a_{0}(980)$ [35]. 


\section{Interpretation of $\sigma, \kappa, f_{0}(980)$ and $a_{0}(980)$}

This is contentious. The front runner appears to be Jaffe's proposal [36] that they are 4-quark states with $S U(3)$ flavour content $3 \otimes \overline{3}$. His scheme explains naturally the progression of their masses: if $f_{0}(980)$ and $a_{0}(980)$ are $s \bar{s}(u \bar{u} \pm d \bar{d})$ states, their near degeneracy is explained naturally, and the large mass difference between $\sigma$ and $a_{0}(980)$. There is support for this interpretation from Lattice QCD calculations, where 4-quark combinations dominate at large $r$ and 2-quark combinations at small $r$ [37]. The decay presumably occurs by fission at small $r$. There is again support for this picture from Ref. [33], where it is shown that the Fourier transform of the $\sigma$ amplitude $\mathrm{v}$, momentum has an RMS radius of $0.4 \mathrm{fm}$.

However, the story cannot be quite so simple. The ratio $r=g^{2}\left(f_{0}(980) \rightarrow\right.$ $K K) / g^{2}\left(a_{0}(980) \rightarrow K K\right)=2.7 \pm 0.5$ disagrees with the ratio 1 predicted from Jaffe's model. The likely explanation is that the pole position of $f_{0}(980)$ is fortuitously very close to the $K K$ threshold, and that of $a_{0}(980)$ lies further away. The $f_{0}(980)$ then necessarily has a large long-range $K K$ cloud, larger than $a_{0}(980)$. Decays to $\pi \pi$ or $\pi \eta$ occur at small $r$ (and some $K K$ ); when this happens, the $K K$ cloud at large $r$ is left 'in the air' (adiabatic approximation). This results in fall-apart decay which is larger for $f_{0}(980)$ than $a_{0}(980)$. This argument accounts for a factor 2 increase in $K K$ decays of $f_{0}(980)$ compared to $a_{0}(980)$, but not the precise pole positions of $f_{0}(980)$ and $a_{0}(980)$.

\section{References}

[1] V.E. Markushin and M.P. Locher, Frascati Physics Series, Vol. XV (1999) 229.

[2] E.M. Aitala et al., Phys. Rev. Lett. 86 (2001) 765.

[3] J.A. Oller, Phys. Rev. D71 (2005) 054030.

[4] M. Ablikim et al., Phys. Lett. B 598 (2004) 149.

[5] S. Weinberg, Phys. Rev. Lett. 17 (1966) 616.

[6] S. Pisluk et al., Phys. Rev. Lett. 87 (2001) 221801. 
[7] B. Hyams et al., Nucl. Phys. B64 (1973) 134.

[8] A.V. Anisovich, V.V. Anisovich and A.V. Sarantsev, Zeit. Phys. A359 (1997) 173.

[9] K.L. Au, D. Morgan and M.R. Pennington, Phys. Rev. D35 (1987) 1633.

[10] G. Colangelo, J. Gasser and H. Leutwyler, Nucl. Phys. B603 (2001) 125.

[11] J.A. Oller and E. Oset, Nucl. Phys. A620 (1997) 438.

[12] J.A. Oller, E. Oset and J.R. Pelaez, Phys. Rev. D59 (1999) 074001.

[13] J.A. Oller and E. Oset, Phys. Rev. D60 (1999) 074023.

[14] M. Jamin, J.A. Oller and A. Pich, Nucl. Phys. B587 (2000) 331.

[15] A. Gomez Nicola and J.R. Pelaez, Phys. Rev. D65 (2002) 054009.

[16] J.R. Pelaez, hep-ph/0307018.

[17] M. Harada, F. Sannino and J. Schechter, Phys. Rev. D54 (1996) 1991.

[18] D. Black et al., Phys. Rev. D58 (1998) 054012.

[19] D. Black, A. H. Fariborz and J. Schechter, Phys. Rev. D61 (2000) 074001.

[20] D. Black et al., Phys. Rev. D64 (2001) 014031.

[21] J. Schechter, hep-ph/0508062.

[22] J. He, Z.G, Xiao and H.Q. Zheng, Phys. Lett. B536 (2002) 59.

[23] H.Q. Zheng, hep-ph/0304173.

[24] H.Q. Zheng et al., Nucl. Phys. A733 (2004) 235.

[25] Z.Y. Zhou et al., JHEP 0502 (2005) 043.

[26] E. van Beveren et al., Z. Phys. C 30 (1986) 615.

[27] E. van Beveren and G. Rupp, Eur. Phys. J. C 22 (2001) 493. 
[28] E.M. Aitala et al, Phys. Rev. Lett. 89 (2002) 121801.

[29] M. Ablikim et al., hep-ex/0506055.

[30] D.V. Bugg, Eur. Phys. J. A24 (2005) 107.

[31] D.Aston et al., Nucl, Phys. B296 )1988) 493.

[32] E.M. Aitala et al., hep-ex/0507099.

[33] D.V. Bugg, Phys. Lett. B572 (2003) 1.

[34] M. Ablikim et al.,Phys. Lett. B607 (2005) 243.

[35] D.V. Bugg, V.V. Anisovich, A.V. Sarantsev and B.S. Zou, Phys. Rev. D50 (1994) 4412.

[36] R.J. Jaffe, Phys. Rev. D15 (1977) 267.

[37] F. Okiharu et al., hep-ph/0507187. 